PROCEEDINGS OF THE

AMERICAN MATHEMATICAL SOCIETY

Volume 127, Number 12, Pages 3671-3676

S 0002-9939(99)05397-6

Article electronically published on July 12, 1999

\title{
THE CONNECTED STABLE RANK OF THE PURELY INFINITE SIMPLE $C^{*}$-ALGEBRAS
}

\author{
YIFENG XUE
}

(Communicated by David R. Larson)

\begin{abstract}
Suppose that $\mathcal{A}$ is a unital purely infinite simple $C^{*}$-algebra. If the class [1] of the unit 1 in $K_{0}(\mathcal{A})$ has torsion, then $\operatorname{csr}(\mathcal{A})=\infty$; if [1] is torsion-free in $K_{0}(\mathcal{A})$, then $\operatorname{csr}(\mathcal{A})=2$. If $\mathcal{A}$ is a non-unital purely infinite simple $C^{*}$-algebra, then $\operatorname{csr}(\mathcal{A})=2$.
\end{abstract}

Before considering the connected stable rank of the purely infinite simple $C^{*}$ algebra, we need to introduce some notation as follows. For the $C^{*}$-algebra $\mathcal{A}$ with unit 1 , we denote by $\mathcal{U}_{n}(\mathcal{A})$ the group of unitary elements in the matrix algebra $\mathrm{M}_{n}(\mathcal{A})$ and also denote by $\mathcal{U}_{n}^{0}(\mathcal{A})$ the connected component of $1_{n}$ in $\mathcal{U}_{n}(\mathcal{A})$. We view $\mathcal{A}^{n}=\left\{\left(a_{1}, \ldots, a_{n}\right)^{T} \mid a_{i} \in \mathcal{A}\right\}$ as the set of all $n \times 1$ matrices over $\mathcal{A}$. For $a=\left(a_{1}, \ldots, a_{n}\right)^{T}$, we set $a^{*}=\left(a_{1}^{*}, \ldots, a_{n}^{*}\right)$-the $1 \times n$ over $\mathcal{A}$. Put

$$
\mathrm{S}_{n}(\mathcal{A})=\left\{\left(a_{1}, \ldots, a_{n}\right)^{T} \in \mathcal{A}^{n} \mid a^{*} a=\sum_{i=1}^{n} a_{i}^{*} a_{i}=1\right\} .
$$

$\mathrm{S}_{n}(\mathcal{A})$ has the base point $e_{n}=(1,0, \ldots, 0)^{T} \in \mathcal{A}^{n}$. Let $\pi_{0}\left(\mathrm{~S}_{n}(\mathcal{A}), e_{n}\right)$ denote the set of all path connected components of $\mathrm{S}_{n}(\mathcal{A})$. We identify the path connected component containing $e_{n}$ with zero element " 0 ". Thus from $[\mathrm{Sr}]$, we have

$$
\operatorname{csr}(\mathcal{A})=\min \left\{n \mid \pi_{0}\left(\mathrm{~S}_{m}(\mathcal{A}), e_{m}\right)=0, \forall m \geq n\right\}
$$

where

$$
\operatorname{csr}(\mathcal{A})=\min \left\{n \mid \mathcal{U}_{m}^{0}(\mathcal{A}) \text { acts transitively on } \mathrm{S}_{m}(\mathcal{A}), \forall m \geq n\right\}
$$

is the connected stable rank of $\mathcal{A}$ defined in [Rf]. If no such integer exists, we set $\operatorname{csr}(\mathcal{A})=\infty$. If $\mathcal{A}$ is not unital, we set $\operatorname{csr}(\mathcal{A})=\operatorname{csr}\left(\mathcal{A}^{+}\right)$, where $\mathcal{A}^{+}$is the $C^{*}$-algebra obtained from $\mathcal{A}$ by adjoining the unit 1 .

Let $p, q$ be two projections in the $C^{*}$-algebra $\mathcal{A}$. We write $p \sim q$ if there is $u \in \mathcal{A}$ such that $p=u^{*} u, q=u u^{*}$ and denote by $[p]$ the equivalence class of $p$ with respect to " $\sim$ " and we also put

$$
D(\mathcal{A})=\{[p] \mid p \text { is a non-zero projection in } \mathcal{A}\} .
$$

Recall that a projection $p$ in $\mathcal{A}$ is called infinite if there is a projection $q$ in $\mathcal{A}$ such that $p \sim q<p$. We say that $\mathcal{A}$ is purely infinite if the closure of $a \mathcal{A} a$ contains an infinite projection for any positive element $a$ in $\mathcal{A}$ (cf. $[\mathrm{Cu}])$.

Received by the editors August 1, 1997 and, in revised form, February 24, 1998.

1991 Mathematics Subject Classification. Primary 46L05.

Key words and phrases. Purely infinite simple $C^{*}$-algebras, connected stable rank, $K$-group of the $C^{*}$-algebras. 
From $[\mathrm{Cu}]$ we have known that if $\mathcal{A}$ is the unital purely infinite simple $C^{*}$ algebra, then $D(\mathcal{A})$ becomes a group with the natural addition $[p]+[q]=\left[p^{\prime}+q^{\prime}\right]$ and $D(\mathcal{A}) \cong K_{0}(\mathcal{A})$ where $p^{\prime} \sim p, q^{\prime} \sim q, p^{\prime} q^{\prime}=0$ and $K_{0}(\mathcal{A})$ is the $K_{0}$-group of $\mathcal{A}$ defined in $[\mathrm{Bk}]$.

Let $p$ be a non-zero projection of the purely infinite simple $C^{*}$-algebra $\mathcal{A}$ with unit 1 . Set

$$
\operatorname{SU}(\mathcal{A})=\left\{u \in \mathcal{A} \mid u^{*} u=1, u u^{*}<1\right\}, \quad \operatorname{SU}_{p}(\mathcal{A})=\left\{u \in S \mathcal{U}(\mathcal{A}) \mid u u^{*} \leq p\right\} .
$$

If $p=1$, then $S \mathcal{U}_{1}(\mathcal{A})=\mathcal{U}(\mathcal{A}) \cup S \mathcal{U}(\mathcal{A})$. In this situation, $S \mathcal{U}_{1}(\mathcal{A})$ is not connected for $\mathcal{U}(\mathcal{A}) \cap S \mathcal{U}(\mathcal{A})=\varnothing$ and $\mathcal{U}(\mathcal{A}), S \mathcal{U}(\mathcal{A})$ are all closed in $S \mathcal{U}_{1}(\mathcal{A})$.

It is known from [Rf, Proposition 6.5] that the topological stable rank of $\mathcal{A}$ is $\infty$ if $\mathcal{A}$ is a purely infinite simple $C^{*}$-algebra. But what is the $\operatorname{csr}(\mathcal{A})$ ? In this paper, we will show that $\operatorname{csr}(\mathcal{A}) \in\{2, \infty\}$. First we have the following known lemma which could be deduced directly from [Cu, Lemma 1.8]:

Lemma 1. Suppose that $\mathcal{A}$ is a purely infinite simple $C^{*}$-algebra with unit 1 . Then for $k \geq 2$ there are isometries $s_{1}, \ldots, s_{k}$ in $\mathcal{A}$ such that $\sum_{i=1}^{k} s_{i} s_{i}^{*}=p$ is a projection in $\mathcal{A}$. Furthermore, if [1] is torsion-free in $K_{0}(\mathcal{A})$ or if $k \not \equiv 1 \bmod n$ when [1] has order $n(1 \leq n<\infty)$ in $K_{0}(\mathcal{A})$, then $[p] \neq[1]$ and if $k \equiv 1 \bmod n$ for above $n$, then $p$ can be chosen as $p=1$.

From Lemma 1, we can choose $k$ isometries $s_{1}, \ldots, s_{k}$ in the unital purely infinite simple $C^{*}$-algebra $\mathcal{A}$ such that $\sum_{i=1}^{k} s_{i} s_{i}^{*}=p$ is a projection in $\mathcal{A}$. Now define a map $\phi_{k}$ of $\mathrm{M}_{k}(\mathcal{A})$ to $\mathcal{A}$ by

$$
\phi_{k}\left(\left(a_{i j}\right)_{k \times k}\right)=\sum_{i, j=1}^{k} s_{i} a_{i j} s_{j}^{*}+1-p .
$$

It is easy to check that $\phi_{k}$ is a $*$-homomorphism with $\phi_{k}\left(1_{k}\right)=1$.

The following corollary somewhat enhances a partial result of [Cu, Lemma 1.8].

Corollary. Let $\mathcal{A}$ be a purely infinite simple $C^{*}$-algebra with unit 1 . Then for any $u \in \mathcal{U}_{k}(\mathcal{A})(k \geq 2)$, there is $u_{0} \in \mathcal{U}_{k}^{0}(\mathcal{A})$ such that $u=u_{0} \operatorname{diag}\left(\phi_{k}(u), 1_{k-1}\right)$.

Proof. Let $s_{1}, \ldots, s_{k}$ be as above and put

$$
X=\left(\begin{array}{cccc}
s_{1} & s_{2} & \ldots & s_{k} \\
0 & 0 & \ldots & 0 \\
\ldots & \ldots & \ldots & \ldots \\
0 & 0 & \ldots & 0
\end{array}\right), \quad Y=\left(\begin{array}{cc}
X & 1_{k}-X X^{*} \\
0 & X^{*}
\end{array}\right) .
$$

Then it is clear that $X^{*} X=1_{k}, Y \in \mathcal{U}_{2 k}(\mathcal{A})$ and moreover, by the definitions of $\phi_{k}$ and $Y$, we have the following identity:

$$
Y \operatorname{diag}\left(u, 1_{k}\right) Y^{*}=\operatorname{diag}\left(\phi_{k}(u), 1_{2 k-1}\right) .
$$

Noting that $Y$ can be decomposed as the form

$$
Y=\left(\begin{array}{cc}
1_{k} & X \\
0 & 1_{k}
\end{array}\right)\left(\begin{array}{cc}
0 & 1_{k}-2 X X^{*} \\
1_{k} & 0
\end{array}\right)\left(\begin{array}{cc}
1_{k} & X^{*} \\
0 & 1_{k}
\end{array}\right)\left(\begin{array}{cc}
1_{k} & 0 \\
-X & 1_{k}
\end{array}\right),
$$

we obtain that $Y \in \mathcal{U}_{2 k}^{0}(\mathcal{A})$. Therefore applying [LZ, Condition (ii)] and [Lin, Lemma 2.2] to (1), we get the assertion.

Let $s_{1}, \ldots, s_{k}$ be as above. Then $\left(s_{1}^{*} u, \ldots, s_{k}^{*} u\right) \in \mathrm{S}_{k}(\mathcal{A})$ for any $u \in S \mathcal{U}_{p}(\mathcal{A})$ because $u u^{*} \leq p$ iff $p u=u$ for $u \in \operatorname{SU}_{p}(\mathcal{A})$. This leads to the following lemma. 
Lemma 2. The map $\beta:\left(\operatorname{SU}_{p}(\mathcal{A}), s_{1}\right) \rightarrow\left(\mathrm{S}_{k}(\mathcal{A}), e_{k}\right)$ given by $\beta(u)=\left(s_{1}^{*} u, \ldots, s_{k}^{*} u\right)$ is homeomorphic.

Proof. Obviously, $\beta$ is continuous and $\beta\left(s_{1}\right)=e_{k}$. Now for any $\left(a_{1}, \ldots, a_{k}\right) \in$ $\mathrm{S}_{k}(\mathcal{A})$, put $u=\sum_{i=1}^{k} s_{i} a_{i}$. Since $s_{i}^{*} s_{j}=\delta_{i j} 1$ and $p s_{i}=s_{i}, i, j=1, \ldots, k$, it follows that $p u=u$ and

$$
u^{*} u=\sum_{i, j} a_{i}^{*} s_{i}^{*} s_{j} a_{j}=\sum_{i=1}^{k} a_{k}^{*} a_{k}=1,
$$

i.e., $u \in \operatorname{SU}_{p}(\mathcal{A})$. Therefore we can define a continuous map $\gamma:\left(\mathrm{S}_{k}(\mathcal{A}), e_{k}\right) \rightarrow$ $\left(S \mathcal{U}_{p}(\mathcal{A}), s_{1}\right)$ by $\gamma\left(\left(a_{1}, \ldots, a_{k}\right)\right)=\sum_{i=1}^{k} s_{i} a_{i}$. By the definitions of $\beta$ and $\gamma$, the assertion follows.

Using Lemma 1 and Lemma 2, we then establish the theorem in the following.

Theorem 1. Suppose that $\mathcal{A}$ is a unital purely infinite simple $C^{*}$-algebra. If the class [1] of the unit 1 in $K_{0}(\mathcal{A})$ has torsion in $K_{0}(\mathcal{A})$, then $\operatorname{csr}(\mathcal{A})=\infty$; if [1] is torsion-free in $K_{0}(\mathcal{A})$, then $\operatorname{csr}(\mathcal{A})=2$.

Proof. We assume that $n(1 \leq n<\infty)$ is the order of [1] in $K_{0}(\mathcal{A})$, i.e., $n$ is the least positive integer such that $n[1]=0$ in $K_{0}(\mathcal{A})$.

If $k \equiv 1 \bmod n$, then $\operatorname{SU}_{1}(\mathcal{A})$ is not connected by Lemma 1 and consequently $\pi_{0}\left(\mathrm{~S}_{k}(\mathcal{A}), e_{k}\right) \neq 0$ by Lemma 2 . If $k \not \equiv 1 \bmod n$, then there are $k$ isometries $s_{1}, \ldots, s_{k}$ in $\mathcal{A}$ such that $\sum_{i=1}^{k} s_{i} s_{i}^{*}=p$ is a projection in $\mathcal{A}$ with $[p] \neq[1]$ by Lemma 1. In this case, we have $u u^{*} \neq p$ for all $u \in S \mathcal{U}_{p}(\mathcal{A})$.

Since $D(\mathcal{A})$ is a group and

$$
[p]=\left[p-u u^{*}\right]+\left[u u^{*}\right]=\left[p-s_{1} s_{1}^{*}\right]+\left[s_{1} s_{1}^{*}\right]
$$

in $D(\mathcal{A})$ for any $u \in S \mathcal{U}_{p}(\mathcal{A})$, it follows that $\left[p-u u^{*}\right]=\left[p-s_{1} s_{1}^{*}\right]$ in $D(\mathcal{A})$. Noting that $u u^{*} \leq p, s_{1} s_{1}^{*} \leq p$, we have $p-u u^{*}, p-s_{1} s_{1}^{*} \in p \mathcal{A} p$ and $p-u u^{*} \sim p-s_{1} s_{1}^{*}$ in $p \mathcal{A} p$. Since $u s_{1}^{*} \in p \mathcal{A} p$ and $u u^{*}=\left(u s_{1}^{*}\right)\left(u s_{1}^{*}\right)^{*}, s_{1} s_{1}^{*}=\left(u s_{1}^{*}\right)^{*}\left(u s_{1}^{*}\right)$ in $p \mathcal{A} p$, it follows that there is $w_{0} \in \mathcal{U}_{1}(p \mathcal{A} p)$ such that $u u^{*}=w_{0}^{*} s_{1} s_{1}^{*} w_{0}$. Put $a=s_{1}^{*} w_{0}^{*} u$ and $w=\left(s_{i}^{*} w_{0} s_{j}\right)_{k \times k}$. Then

$$
u=w_{0} s_{1} a, \quad a \in \mathcal{U}_{1}(\mathcal{A}) \text { and } w \in \mathcal{U}_{k}(\mathcal{A}) .
$$

Since $\left(s_{1}^{*} u, \ldots, s_{k}^{*} u\right)^{T}=w(a, 0, \ldots, 0)^{T}$ by (2), it follows from the Corollary that there exists $u_{0} \in \mathcal{U}_{k}^{0}(\mathcal{A})$ such that

$$
\begin{aligned}
\left(s_{1}^{*} u, \ldots, s_{k}^{*} u\right)^{T} & =u_{0} \operatorname{diag}\left(\phi_{k}(w), 1_{k-1}\right)(a, 0, \ldots, 0)^{T} \\
& =u_{0} \operatorname{diag}\left(w_{0}+1-p, 1_{k-1}\right)(a, 0, \ldots, 0)^{T} \\
& =u_{0} \operatorname{diag}\left(b, b^{*}, 1_{k-2}\right) e_{k},
\end{aligned}
$$

where $b=\left(w_{0}+1-p\right) a \in \mathcal{U}_{1}(\mathcal{A})$, that is, $\left(s_{1}^{*} u, \ldots, s_{k}^{*} u\right)^{T}$ is in the component of $e_{k}$ for $u_{0} \operatorname{diag}\left(b, b^{*}, 1_{k-2}\right) \in \mathcal{U}_{k}^{0}(\mathcal{A})$. Therefore $\pi_{0}\left(\mathrm{~S}_{k}(\mathcal{A}), e_{k}\right)=0$ by Lemma 2 when $k \not \equiv 1 \bmod n$.

The above shows that $\operatorname{csr}(\mathcal{A})=\infty$ when $[1]$ has torsion.

As to the case that [1] is torsion-free in $K_{0}(\mathcal{A})$, we can use Lemma 1 and the same method as in the proof of $\pi_{0}\left(\mathrm{~S}_{k}(\mathcal{A}), e_{k}\right)=0$ when $k \not \equiv 1 \bmod n$ to deduce that $\pi_{0}\left(\mathrm{~S}_{k}(\mathcal{A}), e_{k}\right)=0 \forall k \geq 2$, i.e., $\operatorname{csr}(\mathcal{A}) \leq 2$. Since $\mathcal{A}$ contains an isometry $s_{1}, \operatorname{csr}(\mathcal{A})$ must be equal to two. 
Let $\mathcal{O}_{n}$ be the Cuntz algebra. Then by Theorem 1 , we have $\operatorname{csr}\left(\mathcal{O}_{n}\right)=\infty$ $(2 \leq n<\infty)$, and $\operatorname{csr}\left(\mathcal{O}_{\infty}\right)=2$ since $K_{0}\left(\mathcal{O}_{n}\right)=\mathbb{Z}_{n-1}(n<\infty), K_{0}\left(\mathcal{O}_{\infty}\right)=\mathbb{Z}$ and the class [1] of unit 1 is the generator of $K_{0}\left(\mathcal{O}_{n}\right)(\mathrm{cf} .[\mathrm{Cu}])$.

As an end of this paper, we will consider the $\operatorname{csr}(\mathcal{A})$ if $\mathcal{A}$ is a non-unital purely infinite simple $C^{*}$-algebra. Our result is the following:

Theorem 2. Let $\mathcal{A}$ be a non-unital purely infinite simple $C^{*}$-algebra. Then $\operatorname{csr}(\mathcal{A})$ $=2$.

In order to prove this theorem, we need the following lemmas.

Lemma 3. Let $\mathcal{A}$ be a $C^{*}$-algebra with unit 1 and let $a=\left(a_{1}, \ldots, a_{n}\right)^{T}, b=$ $\left(b_{1}, \ldots, b_{n}\right)^{T} \in \mathrm{S}_{n}(\mathcal{A})$ such that $\left\|a_{i}-b_{i}\right\| \leq\left(\sum_{i=1}^{n}\left\|a_{i}\right\|\right)^{-1}$. Then $a, b$ are in the same component of $\mathrm{S}_{n}(\mathcal{A})$.

Proof. Put $c_{t}=((1-t) a+t b)^{*}((1-t) a+t b) \forall t \in[0,1]$. Since $a^{*} a=b^{*} b=1$, it follows that

$$
\left\|1-c_{t}\right\|=\left\|(1-t) t\left(a^{*}(b-a)+(b-a)^{*} a\right)\right\| \leq \frac{1}{2} \quad \forall t \in[0,1] .
$$

Set $G_{t}=((1-t) a+t b)\left(c_{t}\right)^{-1 / 2}$. Then $G_{t}$ is the path from $a$ to $b$ in $\mathrm{S}_{n}(\mathcal{A})$.

Lemma 4. Suppose that $\mathcal{A}$ is a purely infinite simple $C^{*}$-algebra. Then there exists a in $\mathcal{A}$ with $0 \leq a<1$ such that $\overline{a \mathcal{A} a}$ is non-unital.

Proof. Choose a non-trivial projection $q$ in $\mathcal{A}$. Since $\mathcal{A}$ is purely infinite simple, there is a projection $q_{0}$ in $\mathcal{A}$ such that $q_{0}<q$ and $q_{0} \sim q$. Using the same method as in the proof of [Cu, Lemma 1.8], we can find a sequence of pairwise orthogonal projections $r_{i}<q-r_{0}$ in $q \mathcal{A} q$ such that $r_{i} \sim q, i \geq 0$. Let $\left\{\beta_{n}\right\}_{0}^{\infty}$ be a sequence in $(0,1)$ with $\lim _{n \rightarrow \infty} \beta_{n}=0$. Set $a=\sum_{n=0}^{\infty} \beta_{n} r_{n}$. Then $a \in \mathcal{A}$ and $0 \leq a<1$.

We now claim that $\overline{a \mathcal{A} a}$ is non-unital for such $a$. If the statement is not true, then there is a projection $R$ in $\overline{a \mathcal{A} a}$ such that $R$ is the unit of $\overline{a \mathcal{A} a}$. Thus we have $R a^{3}=a^{3}$ and $\|R=a x a\|<\frac{1}{2}$ for some $x \in \mathcal{A}$. Consequently, $r_{n} \leq R$ and

$$
\left\|r_{n}-\beta_{n}^{2} r_{n} x r_{n}\right\| \leq\left\|r_{n}(R-a x a) r_{n}\right\|<\frac{1}{2} .
$$

This implies that $\beta_{n}^{2}\|x\| \geq \beta_{n}^{2}\left\|r_{n} x r_{n}\right\|>\left\|r_{n}\right\|-\frac{1}{2}=\frac{1}{2}$ which is contrary to the assumption that $\lim _{n \rightarrow \infty} \beta_{n}=0$.

Proof of Theorem 2. If $\mathcal{A}$ is a $\sigma$-unital, then by [Zh, Theorem 1.2] $\mathcal{A}$ is stable, i.e., $\mathcal{A} \cong \mathcal{A} \otimes \mathcal{K}$, where $\mathcal{K}$ is the algebra of all compact operators on a separable, infinite dimensional Hilbert space over $\mathbb{C}$. In this case, $\operatorname{csr}(\mathcal{A}) \leq 2$ by [Ni, Corollary 2.5].

Consider the general case. For $k \geq 2$, let $\tilde{a}=\left(a_{1}+\lambda_{1}, \ldots, a_{k}+\lambda_{k}\right)^{T} \in \mathrm{S}_{k}\left(\mathcal{A}^{+}\right)$ where $a_{i} \in \mathcal{A}, \lambda_{i} \in \mathbb{C}$. Set

$$
x=\sum_{i=1}^{k}\left(a_{i}^{*} a_{i}+a_{i} a_{i}^{*}\right) .
$$

Then for $\varepsilon=\frac{1}{2} \min \left((6 k)^{-1},(1+12 k)^{-1}\left(\sum_{i=1}^{n}\left\|a_{i}+\lambda_{i}\right\|\right)^{-1}\right)$, there is a projection $r$ in $\mathcal{A}$ such that $\|x(1-r)\|<\varepsilon^{2}$ by [LZ, Condition (ii)] and [BP, Theorem 2.6]. Since $(1-r) \mathcal{A}(1-r)$ is purely infinite simple ([Zh, Theorem 1.3]), there is by Lemma 4 a $c$ in $(1-r) \mathcal{A}(1-r)$ with $0 \leq c<1-r$ such that $\overline{c \mathcal{A} c}$ is non-unital. Put $d=r+c$. 
Then $0 \leq d \leq 1$ and $\mathcal{B}=\overline{d \mathcal{A d}}$ is non-unital (if $Q$ is the unit of $\mathcal{B}, Q-r$ must be the unit of $\overline{c \mathcal{A} c}$ ) and furthermore, we have

$$
x^{1 / 2}(1-d)^{2} x^{1 / 2} \leq x^{1 / 2}(1-d) x^{1 / 2} \leq x^{1 / 2}(1-r) x^{1 / 2} \leq \varepsilon^{2} .
$$

Now (3) indicates that $\left\|a_{i}(1-d)\right\|<\varepsilon$ and $\left\|(1-d) a_{i}\right\|<\varepsilon, 1 \leq i \leq k$. Set $b_{i}=d a_{i} d+\lambda_{i}, b=\sum_{i=1}^{k} b_{i}^{*} b_{i}$. Then $b_{i}, b \in \mathcal{B}^{+},\left\|a_{i}+\lambda_{i}-b_{i}\right\|<2 \varepsilon, 1 \leq i \leq k$, and

$$
\begin{aligned}
\|1-b\| & =\left\|\sum_{i=1}^{k}\left(a_{i}+\lambda_{i}-b_{i}\right)^{*}\left(a_{i}+\lambda_{i}\right)+\sum_{i=1}^{k} b_{i}^{*}\left(a_{i}+\lambda_{i}-b_{i}\right)\right\| \\
& \leq 3 \sum_{i=1}^{k}\left\|a_{i}+\lambda_{i}-b_{i}\right\|<6 k \varepsilon<\frac{1}{2}
\end{aligned}
$$

(for $\left\|a_{i}+\lambda_{i}\right\| \leq 1,\left\|\lambda_{i}\right\| \leq 1$ and $\left.\left\|b_{i}\right\|=\left\|d\left(a_{i}+\lambda_{i}\right) d+\lambda_{i}\left(1-d^{2}\right)\right\| \leq 2\right)$. Thus $b$ is invertible in $\mathcal{B}^{+}$with $\left\|b^{-1}\right\|<2$. Since $b>0$, we get that $\left\|b^{-1 / 2}\right\|<2^{1 / 2}$ and

$$
\left\|1-b^{-1 / 2}\right\|=\left\|b^{-1 / 2}\left(1-b^{1 / 2}\right)\right\|<2^{1 / 2}\|1-b\|<12 k \varepsilon .
$$

Now set $c_{i}=b_{i} b^{-1 / 2} \in \mathcal{B}^{+}$. Then $\left(c_{1}, \ldots, c_{k}\right)^{T} \in \mathrm{S}_{k}\left(\mathcal{B}^{+}\right)$and

$$
\begin{aligned}
\left\|a_{i}+\lambda_{i}-c_{i}\right\| & =\left\|a_{i}+\lambda_{i}-b_{i}+b_{i}\left(1-b^{-1 / 2}\right)\right\| \\
& <2 \varepsilon+24 k \varepsilon \leq\left(\sum_{i=1}^{k}\left\|a_{i}+\lambda_{i}\right\|\right)^{-1} .
\end{aligned}
$$

Since $\mathcal{B}$ is a non-unital, $\sigma$-unital, hereditary $C^{*}$-subalgebra of $\mathcal{A}$, it follows from [Zh, Theorem 1.2] that $\mathcal{B}$ is stable and consequently, by means of the above argument, there exists $w \in \mathcal{U}_{k}^{0}\left(B^{+}\right) \subset \mathcal{U}_{k}^{0}\left(\mathcal{A}^{+}\right)$such that $\left(c_{1}, \ldots, c_{k}\right)^{T}=w e_{k}$. Applying Lemma 3 to (4), we obtain that $\tilde{a}$ is in the component containing $e_{k}$. Thus $\operatorname{csr}\left(\mathcal{A}^{+}\right) \leq 2$. Now choose an isometry $s$ in $r \mathcal{A} r$ with $s^{*} s=r, s s^{*}<r$. Set $T=s+1-r$. Then $T$ is an isometry in $\mathcal{A}^{+}$. Therefore $\operatorname{csr}(\mathcal{A})=2$.

\section{ACKNOWLEDGEMENT}

The author wishes to thank Professor Huaxin Lin for his helpful suggestions to the proofs of Theorem 1 and Theorem 2.

\section{REFERENCES}

[Bk] B. Blackadar, K-Theory for Operator Algebras, MSRI Publications No. 5, SpringerVerlag/New York/Berlin/Heidelberg/London/Paris/Tokyo, 1986. MR 88g:46082

[BP] L. G. Brown and G. K. Pedersen, $C^{*}$-algebras of real rank zero, J. Funct. Anal. 99 (1991), 131-149. MR 92m:46086

[Cu] J. Cuntz, K-Theory for Certain $C^{*}$-Algebras, Ann. of Math. 113 (1981), 181-197. MR 84c: 46058

[Lin] H. Lin, Approximation by normal elements with finite spectra in $C^{*}$-algebras of real rank zero, Pacific J. Math. 173 (1995), 443-489. MR 98h:46059

[LZ] H. Lin and S. Zhang, On infinite simple $C^{*}$-algebras, J. Funct. Anal. 100 (1991), 221-231. MR 92m: 46088

[Ni] V. Nistor, Stable range for tension products of extensions of $\mathcal{K}$ by $C(X)$, J. Operator Theory 16 (1986), 387-396. MR 88b:46085 
[Rf] M. A. Rieffel, Dimensional and stable rank in the $K$-theory of $C^{*}$-Algebras, Proc. London Math. Soc. 46, no. (3) (1983), 301-333. MR 84g:46085

[Sr] H. Schröder, On the homotopy type of the regular group of a $W^{*}$-algebra, Math. Ann. 167 (1992), 171-277.

[Zh] S. Zhang, $C^{*}$-algebras with real rank zero and their multiplier algebras, I, Pacific J. Math. 155 (1992), 169-197.

Institute of Fundamental Education, East China University of Science and Technology, Shanghai 200237, People's Republic of China

Current address: Department of Mathematics, East China University of Science and Technology, Shanghai 200237, People's Republic of China 